\title{
LOCALLY CONTRACTIBLE SPACES THAT ARE ABSOLUTE NEIGHBORHOOD RETRACTS ${ }^{1}$
}

\author{
WILLIAM E. HAVER
}

\begin{abstract}
It is shown that every locally contractible metric space that is the countable union of finite dimensional compacta is an absolute neighborhood retract.
\end{abstract}

The theorem of this note was motivated by a desire to show that the space of all piecewise linear homeomorphisms of a compact piecewise linear manifold onto itself (which Geoghegan [4] has shown to be the countable union of finite dimensional compacta) is an ANR. This result follows as a corollary to the theorem of this paper. The author wishes to thank William Mason for helpful conversations.

In the proof of the theorem we shall use the fact that every open covering $\alpha$ of a metric space, $D$, has an open barycentric refinement, $\beta$ (i.e. an open covering with the property that if $x \in D$ then there exists $U \in \alpha$ such that if $x \in V \in \beta$, then $V \subset U$ ). We shall also use the fact that every open covering $\alpha$ of a separable metric space, $D$, has a countable star-finite refinement, $\beta$ (i.e. a countable open covering with the property that given $U \in \beta$ there exists a $V \in \alpha$ such that $U \subset V$ and $\{W \in \beta \mid W \cap U \neq \varnothing\}$ is finite). These facts are well known and can be found, for example, in Dugundji [1]. We start with the proof of the following Technical Lemma which is stated using terminology that will be helpful in the proof of the theorem.

TeChnical Lemma. Let $D=\bigcup_{k=1}^{\infty} D_{k}$ be a metric space where each $D_{k}$ is nonempty, compact and finite dimensional. Let $\left\{\varepsilon_{i}\right\}_{i=1}^{\infty}$ be a sequence of positive numbers. Then for each positive integer, $n$, there is a sequence of collections of open sets, $\mathscr{U}_{n, n}, \mathscr{U}_{n, n+2}, \mathscr{U}_{n, n+4}, \cdots$ with the following properties:

(a) if $U_{n, j} \in \mathscr{U}_{n, j}$, then $\operatorname{diam} U_{n, j}<\varepsilon_{j}$,

(b) if $U_{n, j}, U_{n, j}^{\prime} \in \mathscr{U}_{n, j}$, then $U_{n, j} \cap U_{n, j}^{\prime}=\varnothing$,

(c) if $U_{n, j} \in \mathscr{U}_{n, j}$, there is an integer $k \leqq j$ so that $D_{k} \cap U_{n, j} \neq \varnothing$,

(d) for each $n, \mathscr{U}^{n}=\bigcup_{i=0}^{\infty} \mathscr{U}_{n, n+2 i}$ is a countable cover of $D$.

Received by the editors July 9, 1972 and, in revised form, December 6, 1972.

AMS (MOS) subject classifications (1970). Primary 54C55; Secondary 58D15.

Key words and phrases. ANR, locally contractible, spaces of piecewise linear homeomorphisms.

${ }^{1}$ Research supported in part by NSF Grant GP 33872.

(c) American Mathematical Society 1973 
Proof. Fix an integer $n$ and let $d_{k}=\left(\operatorname{dim} D_{k}\right)+1$. Then for each $k, D_{k}$ can be written as the union of $d_{k} 0$-dimensional subsets of $D_{k}$ which we shall label in the following manner.

$$
D_{k}=X_{n, n+2} \sum_{i=1}^{k-1} d_{i} \cup X_{n, n+2} \sum_{i=1}^{k-1} d_{i}+2 \cup \cdots \cup X_{n, n+2} \sum_{i=1}^{k-1} d_{i}+2\left(d_{k}-1\right) .
$$

Since each such set, $X_{n, j}$, is a 0 -dimensional subset of $D_{k}$, there exists a pairwise disjoint collection of nonempty $X_{n, j}$-open sets, $\mathscr{W}_{n, j}$, such that for each $W_{n, j} \in \mathscr{W}_{n, j}$ the diameter of $W_{n, j}$ is less than $\varepsilon_{j} / 3$ and $\bigcup_{W_{n, j} \in \mathscr{W}_{n, j}} W_{n, j}=X_{n, j}$

For each $W_{n, j} \in \mathscr{W}_{n, j}$, let

$$
U_{n, j}=\left\{x \in D \mid d\left(x, W_{n, j}\right)<\min \left(\frac{\varepsilon_{j}}{3}, d\left(x, \bigcup_{W_{n^{\prime}, j}^{\prime} \in W_{n, j} ; W_{n, j}^{\prime} \neq W_{n, j}} W_{n, j}^{\prime}\right)\right)\right\} .
$$

Let $\mathscr{U}_{n, j}=\left\{U_{n, j} \mid W_{n, j} \in \mathscr{W}_{n, j}\right\}$.

Property (a) is met trivially by the definition of each $U_{n, j}$. If $U_{n, j} \neq U_{n, j}^{\prime}$ are elements of $\mathscr{U}_{n, j}$ and $x \in U_{n, j} \cap U_{n, j}^{\prime}$, then $d\left(x, W_{n, j}\right)<d\left(x, W_{n, j}^{\prime}\right)$ and $d\left(x, W_{n, j}^{\prime}\right)<d\left(x, W_{n, j}\right)$. Hence property (b) is satisfied. If $U \in \mathscr{U}_{n, j}$ then $U \cap X_{n, j} \neq \varnothing$. But $X_{n, j} \subset D_{k}$ for some $k$ and since $d_{i} \geqq 1$ for each $i, k \leqq j$. Therefore, condition (c) is satisfied. Condition (d) is also met since for each $k, D_{k}$ is covered by

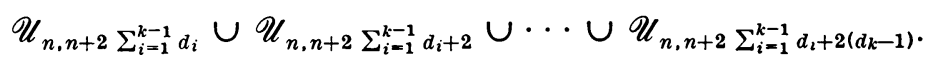

Since $D$ is separable there is a countable subcover of $\mathscr{U}^{n}$ which still satisfies conditions (a)-(d).

Let $\alpha$ be an open covering of $D$. A homotopy $H: D \times I \rightarrow D$ is limited by $\alpha$ if for each $x \in D$ there is an element $U$ of $\alpha$ such that $H(x, t) \in U$ for all $t \in I$. A space $P \alpha$-dominates $D$ if there are maps $b: D \rightarrow P$ and $f: P \rightarrow D$ and a homotopy $H: D \times I \rightarrow D$, limited by $\alpha$, such that for each $x \in D, H(x, 0)=$ $f b(x)$ and $H(x, 1)=x$.

In the following theorem we shall show that for every open covering $\alpha$ of $D$ there is a locally finite polyhedron, $P, \alpha$-dominating $D$. Hence, by a theorem of Hanner [5] (cf. Hu [6, p. 139]), D is an ANR.

THEOREM. Let $D=\bigcup_{k=1}^{\infty} D_{k}$ be a locally contractible metric space where $D_{k}$ is a compact finite dimensional space for each $k$. Then $D$ is an ANR.

Proof. Let $\alpha$ be an open cover of $D$ and $\beta$ an open barycentric refinement of $\alpha$. For each natural number $i$, let $\eta_{i}>0$ be chosen so that if $x \in \bigcup_{k=1}^{i} D_{k}$, then $N_{\eta_{i}}(x) \subset U$ for some $U \in \beta$. We now define a decreasing 
sequence of positive numbers $\left\{\varepsilon_{i}\right\}_{i=1}^{\infty}$ with the following properties:

(i) $\varepsilon_{i} \leqq \min \left(1 / 2(i+1), \eta_{i+1}\right)$,

(ii) if $k \leqq i$ and $x \in D_{k}$, then $N_{3_{i} \varepsilon}(x)$ is contractible to $x$ in $N_{\varepsilon_{i-1}}(x)$.

By the Technical Lemma there exists for each positive integer, $n$, a sequence of collections of open sets $\mathscr{U}_{n, n}, \mathscr{U}_{n, n+2}, \mathscr{U}_{n, n+4}$, satisfying properties (a)-(d).

Next we define an open cover, $\gamma$, of $D \times[0,1)$ as follows. Let

$$
\gamma=\left\{U \times[0,1 / 2) \mid U \in \mathscr{U}^{1}\right\} \cup \bigcup_{n=2}^{\infty}\left\{U \times\left(\frac{2^{n}-3}{2^{n}}, \frac{2^{n}-1}{2^{n}}\right) \mid U \in \mathscr{U}^{n}\right\} .
$$

An arbitrary element of $\gamma$ is of the form $U \times J$, where $U \in \mathscr{U}^{n}$ for some $n$ and $J$ is an open subinterval of $[0,1)$. Note that if $U_{n, j} \times J$ and $U_{m, k} \times K$ are elements of $\gamma$ with nonempty intersection, then $j \neq k$. (Throughout the following $U_{n, j}$ will denote an element of $\mathscr{U}_{n, j}$.)

Let $\zeta$ be a countable star-finite refinement of $\gamma, \mathscr{P}$ the nerve of $\zeta$ and $B: D \times[0,1) \rightarrow \mathscr{P}$ the standard barycentric map. Note that $\mathscr{P}$ is a locally finite polyhedron. For each $Z \in \zeta$ pick a pair $(n, j)$ such that for some $U_{n, j} \times J \in \gamma, Z \subset U_{n, j} \times J$. Set $\phi(Z)=U_{n, j} \times J$ and by extending linearly define a map $\phi$ from $\mathscr{P}$ into the nerve of $\gamma$. Let $\phi(\mathscr{P})=Q \cdot Q$ is a (not necessarily locally finite) polyhedron.

We will define a map $G: Q \rightarrow D$ inductively on the $m$-skeletons, $Q^{m}$, of $Q$. For each vertex $U_{n, j} \times J$ of $Q$, let $G_{0}\left(U_{n, j} \times J\right)$ be an element of $U_{n, j} \cap D_{k}$ for some $k \leqq j$ and let $\psi_{n, j}: N_{3 \varepsilon_{j}}\left(G_{0}\left(U_{n, j} \times J\right)\right) \times[0,1] \rightarrow N_{\varepsilon_{j-1}}\left(G_{0}\left(U_{n, j} \times J\right)\right)$ be a fixed homotopy with the property that $\psi_{n, j}(x, 0)=x$ and $\psi_{n, j}(x, 1)=$ $G_{0}\left(U_{n, j} \times J\right)$ for each $x \in N_{3 \varepsilon_{j}}\left(G_{0}\left(U_{n, j} \times J\right)\right)$. [The choice of $G_{0}\left(U_{n, j} \times J\right)$ is made possible by property (c) of the lemma and the existence of $\psi_{n, j}$ is guaranteed by property (ii) of the sequence $\left\{\varepsilon_{i}\right\}_{i=1}^{\infty}$. ]

We have thereby defined $G_{0}: Q^{0} \rightarrow D$. If $\sigma=\left\langle U_{n_{0}, j_{0}} \times J_{0}, U_{n_{1}, j_{1}} \times J_{1}\right\rangle$ is a simplex in the 1-skeleton of $Q$ and $j_{0}<j_{1}$, define $G_{1} \mid \sigma$ by considering $\sigma$ as the cone on $U_{n_{1}, j_{1}} \times J_{1}$ with vertex $U_{n_{0}, j_{0}} \times J_{0}$ and making use of $\psi_{n_{0}, j_{0}} \mid G_{0}\left(U_{n_{1}, j_{1}} \times J_{1}\right) \times I$ in a canonical manner. Note that $\psi_{n_{0}, j_{0}}$ is defined on $G_{0}\left(U_{n_{1}, j_{1}} \times J_{1}\right) \times I$ since $\sigma \in$ nerve of $\gamma$ implies that $\left(U_{n_{0}, j_{0}} \times J_{0}\right) \cap$ $\left(U_{n_{1}, j_{1}} \times J_{1}\right) \neq \varnothing$ and $\operatorname{diam}\left(U_{n_{0}, j_{0}}\right)<\varepsilon_{j_{0}}$ and $\operatorname{diam}\left(U_{n_{1}, j_{1}}\right)<\varepsilon_{j_{1}}<\varepsilon_{j_{0}}$.

Assume inductively that for each $r<m, G_{r}: Q^{r} \rightarrow D$ has been defined for each $r$-simplex $\sigma=\left\langle U_{n_{0}, j_{0}} \times J_{0}, U_{n_{1}, j_{1}} \times J_{1}, \cdots, U_{n_{r}, j_{r}} \times J_{r}\right\rangle$ (where $j_{0}<j_{k}$, $k=1, \cdots, r)$ by considering $\sigma$ as the cone on $\left\langle U_{n_{1}, j_{1}} \times J_{1}, \cdots, U_{n_{r}, j_{r}} \times J_{r}\right\rangle$ with vertex $U_{n_{0}, j_{0}} \times J_{0}$ and making use of $\psi_{n_{0}, j_{0}} \mid G_{r-1}\left(\left\langle U_{n_{1}, j_{1}}, \cdots, U_{n_{r}, j_{r}}\right\rangle\right) \times I$, that $G_{r} \mid Q^{r-1}=G_{r-1}$ and that $G_{r}$ restricted to any locally finite subcomplex of $Q^{r}$ is continuous.

Now, let $\tau=\left\langle U_{n_{0}, j_{0}} \times J_{0}, U_{n_{1}, j_{1}} \times J_{1}, \cdots, U_{n_{m}, j_{m}} \times J_{m}\right\rangle$ be a simplex of $Q^{m}$. Since $\tau$ is a simplex of $Q, \bigcap_{k=0}^{m}\left(U_{n_{k}, j_{k}} \times J_{k}\right) \neq \varnothing$ and hence $j_{l} \neq j_{k}$ if $l \neq k$. Therefore, without loss of generality, assume that $j_{0}<j_{1}<j_{k}$, for $k=2, \cdots, m$. By the inductive hypothesis and properties of $\psi_{n_{1}, j_{1}}$ we know 
that $G_{m-1}\left(\left\langle U_{n_{1}, j_{1}} \times J_{1}, \cdots, U_{n_{m}, j_{m}} \times J_{m}\right\rangle\right)$ is contained in the $\varepsilon_{j_{1}-1}$ and hence the $\varepsilon_{j_{0}}$ neighborhood of $G_{0}\left(U_{n_{1}, j_{1}}^{m} \times J_{1}\right)$. The diameters of $U_{n_{0}, j_{0}}$ and $U_{n_{1}, j_{1}}$ are each less than $\varepsilon_{j_{0}}$, so

$$
G_{m-1}\left(\left\langle U_{n_{1}, j_{1}} \times J_{1}, \cdots, U_{n_{m}, j_{m}} \times J_{m}\right\rangle\right) \subset N_{3 \varepsilon_{0}}\left(G_{0}\left(U_{n_{0}, j_{0}} \times J_{0}\right)\right) .
$$

Now, consider $\tau$ as the cone on $\left\langle U_{n_{1}, j_{1}} \times J_{1}, \cdots, U_{n_{m}, j_{m}} \times J_{m}\right\rangle$ with vertex $U_{n_{0}, j_{0}} \times J_{0}$ and define $G_{m} \mid \tau$ in a canonical manner by making use of

$$
\psi_{n_{0}, j_{0}} \mid G_{m-1}\left(\left\langle U_{n_{1}, j_{1}} \times J_{1}, \cdots, U_{n_{m}, j_{m}} \times J_{m}\right\rangle\right) \times I .
$$

We thus define $G_{m}: Q^{m} \rightarrow D$ and note that $G_{m}$ restricted to any locally finite subcomplex of $Q$ is continuous and that $G_{m} \mid Q^{m-1}=G_{m-1}$.

For each $m$, define $F_{m}: \mathscr{P}^{m} \rightarrow D$ by $F_{m}(x)=G_{m}(\phi(x))$. Hence $\lim _{m \rightarrow \infty} F_{m}=$ $F: \mathscr{P} \rightarrow D$ is continuous by the continuity of each $F_{m}$ and the local finiteness of $\mathscr{P}$.

Let $b: D \rightarrow \mathscr{P}$ be defined by $b(x)=B(x, 0)$ and $P=b(D)$. Then $P$ is a locally finite polyhedron naturally included in $\mathscr{P}$. Let $f: P \rightarrow D$ be the restriction of $F$ to $P$. To complete the proof of the theorem it must be shown that $f b$ is homotopic to the identity map through a homotopy limited by $\alpha$. To show this we consider the homotopy $H: D \times I \rightarrow D$ defined by

$$
\begin{aligned}
H(x, t) & =F B(x, t), & & t \neq 1, \\
& =x, & & t=1 .
\end{aligned}
$$

If $H$ is continuous it provides a homotopy between $f b$ and $\mathrm{id}_{D}$. For $(x, t) \in D \times I$ and $t \neq 1, H$ is continuous by the continuity of $B$ and $F$.

Let $(x, t) \in D \times[0,1)$ and let $U_{n, j} \times J$ be the unique element of $\gamma$ with the following properties:

(1) There exists a $Z \in \zeta$ such that $(x, t) \in Z$ and $\phi(Z)=U_{n, j} \times J$.

(2) If $(x, t) \in Z^{\prime} \in \zeta$ and $\phi\left(Z^{\prime}\right)=U_{m, k} \times K$, then $k>j$.

We note that $\phi B(x, t)$ is contained in a simplex $\sigma$ of $Q$ such that $\sigma=$ $\left\langle U_{n, j} \times J, U_{n_{1}, j_{1}} \times J_{1}, \cdots, U_{n_{r}, j_{r}} \times J_{r}\right\rangle$ where $j<j_{i}(i=1, \cdots, r)$ and hence that $H(x, t)=F B(x, t) \in N_{\varepsilon_{j-1}}\left(G_{0}\left(U_{n, j} \times J\right)\right)$. Now, by the definition of $G_{0}$, there is an integer $k \leqq j$ so that $G_{0}\left(U_{n, j} \times J\right) \in D_{k} \cap U_{n, j}$. Since $\operatorname{diam}\left(U_{n, j}\right)<$ $\varepsilon_{j}, x \in N_{\varepsilon_{j}}\left(G_{0}\left(U_{n, j} \times J\right)\right) \subset N_{\varepsilon_{j-1}}\left(G_{0}\left(U_{n, j} \times J\right)\right)$. But by the definition of the sequence $\left\{\varepsilon_{i}\right\}_{i=1}^{\infty}$ this implies that there is some ${ }_{(x, t)} U \in \beta$ so that $\{x\} \cup$ $\{F B(x, t)\} \subset N_{\varepsilon_{j-1}}\left(G_{0}\left(U_{n, j} \times J\right)\right) \subset N_{\eta_{j}}\left(G_{0}\left(U_{n, j} \times J\right)\right) \subset(x, t) U$. Pick $A \in \alpha$ such that $\operatorname{St}_{\beta}(x) \subset A$. Then $H(x, I) \subset A$ and $H$ is limited by $\alpha$.

To see that $H$ is continuous we note that if $t \in\left(\left(2^{n}-3\right) / 2^{n},\left(2^{n}-1\right) / 2^{n}\right)$, $d(x, F B(x, t)) \leqq d\left(x, G_{0}\left(U_{n, j} \times J\right)\right)+d\left(G_{0}\left(U_{n, j} \times J\right), F B(x, t)\right)<\operatorname{diam}\left(U_{n, j}\right)+$ $\varepsilon_{j-1}<\varepsilon_{j}+\varepsilon_{j-1}<\varepsilon_{n}+\varepsilon_{n-1}<2 \varepsilon_{n-1}<2(1 / 2(n+1-1))<1 / n$.

COROllary. The space, $\mathrm{PLH}(M)$, of all piecewise linear homeomorphisms of a compact piecewise linear manifold, $M$, onto itself is an ANR.

Proof. As stated in the introduction, Geoghegan [4] has shown that $\operatorname{PLH}(M)$ is the countable union of compact finite dimensional sets. 
Robert Edwards [private communication] has shown that Cernavskir's meshing argument ([2], [3]) can be modified to show that $\operatorname{PLH}(M)$ is locally contractible at 1 , the identity homeomorphism on $M$, and hence locally contractible. To obtain this result Edwards first shows that given any $\varepsilon>0$ there is a $\delta>0$ such that for any $\eta>0$, there is a deformation $\Phi: N(1, \delta) \times I \rightarrow N(1, \varepsilon)$ of the $\delta$-neighborhood of 1 in $H(M)$ into the $\eta$ neighborhood of 1 such that for any $h \in N(1, \delta) \cap \operatorname{PLH}(M), \Phi(h, t)$ is a PL isotopy of $h$. (As in Cernavskii it suffices to prove a handle version of this statement. But this follows immediately from Cernavskiřs proof of his fundamental lemma [2], [3] by stopping the meshing process once $N(1, \delta)$ has been deformed into $N(1, \eta)$. This occurs after a finite number of steps and since all auxiliary homeomorphisms and pushes can be chosen to be PL, the deformation will produce PL isotopies from PL homeomorphisms.) Therefore $\operatorname{PLH}(M)$ is seen to be locally contractible at the identity by stacking a countable number of such deformations end to end so that in the limit they converge to the constant map sending $N(1, \delta)$ to 1 .

Some questions. If $K$ is a finite simplicial complex, Geoghegan's result shows that $\operatorname{PLH}(K)$ is the countable union of compact finite dimensional sets. Is $\operatorname{PLH}(K)$ locally contractible and hence an ANR? Mason and Luke ([7], [8]) have shown that the space of all homeomorphisms on a compact 2-manifold is an ANR. Is the space of all homeomorphisms on a compact manifold an ANR?

\section{BIBLIOGRAPHY}

1. J. Dugundji, Topology, Allyn and Bacon, Boston, Mass., 1966. MR 33 \#1824.

2. A. V. Cernavskiǐ, Local contractibility of the homeomorphism group of a manifold, Dokl. Akad. Nauk SSSR 182 (1968), 510-513=Soviet Math. Dokl. 9 (1968), 1171-1174. MR 38 \#5241.

3. - Local contractibility of the homeomorphism group of a manifold, Mat. Sb. (121) 79 (1969), 307-356. (Russian) MR 41 \#4554.

4. R. Geoghegan, On spaces of homeomorphisms, embeddings and functions. II: The piecewise linear case, Proc. London Math. Soc. (to appear).

5. O. Hanner, Some theorems on absolute neighborhood retracts, Ark. Mat. 1 (1951), 389-408. MR 13, 266.

6. S. Hu, Theory of retracts, Wayne State Univ. Press, Detroit, Mich., 1965. MR 31 \# 6202.

7. R. Luke and W. K. Mason, The space of homeomorphisms on a compact twomanifold is an absolute neighborhood retract, Trans. Amer. Math. Soc. 164 (1972), 275286.

8. W. K. Mason, The space of all self-homeomorphisms of a 2-cell which fix the cell's boundary is an absolute retract, Trans. Amer. Math. Soc. 161 (1971), 185-205. MR 44 \#3283.

Department of Mathematics, University of Tennessee, Knoxville, Tennessee 37916 